Relations industrielles

Industrial Relations

\title{
La double personnalité d'un concierge!
}

\section{Fernand Morin}

Volume 41, numéro 4, 1986

URI : https://id.erudit.org/iderudit/050263ar

DOI : https://doi.org/10.7202/050263ar

Aller au sommaire du numéro

Éditeur(s)

Département des relations industrielles de l'Université Laval

ISSN

0034-379X (imprimé)

1703-8138 (numérique)

Découvrir la revue

Citer cet article

Morin, F. (1986). La double personnalité d'un concierge! Relations industrielles /

Industrial Relations, 41(4), 835-839. https://doi.org/10.7202/050263ar
Résumé de l'article

La double personnalite d'un concierge!
Tous droits réservés (c) Département des relations industrielles de l'Université Laval, 1986
Ce document est protégé par la loi sur le droit d'auteur. L’utilisation des services d'Érudit (y compris la reproduction) est assujettie à sa politique d'utilisation que vous pouvez consulter en ligne.

https://apropos.erudit.org/fr/usagers/politique-dutilisation/ 


\section{DROIT DU TRAVAIL}

\section{La double personnalité d'un concierge!}

\section{Fernand Morin}

Le Tribunal du travail vient de qualifier d'entrepreneur un travailleur préposé à l'entretien ménager puisqu'il était lié au donneur d'ouvrage par le

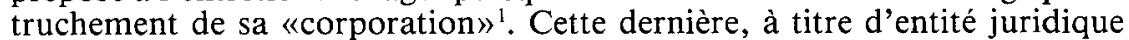
distincte, servit d'écran suffisamment opaque pour cacher le travailleur qui s'y trouvait et qui était à la fois président de sa "corporation» et principal salarié d'exécution. Il semble qu'il faille savoir distinguer cette «réalité contractuelle» des autres réalités pour saisir la subtilité d'une pareille situation. $\hat{A}$ cette fin, il convient de rappeler succintement les principaux faits de l'affaire et les motifs du tribunal.

\section{LES FAITS}

Il s'agit d'un milieu où un syndicat est accrédité, depuis 1976, pour représenter les concierges de la commission scolaire. Le travail ménager de vingt-cing écoles de l'employeur est effectué par des salariés-concierges. Cependant, dès qu'un concierge quitte, on procède à un appel d'offre pour obtenir les services d'un entrepreneur pour effectuer l'ensemble des travaux ménagers en une école ou pour une partie d'une école. Lors des événements en cause, le travail ménager de dix écoles est effectué par de tels entrepreneurs et en deux de ces écoles, les tâches sont réparties entre un entrepreneur et quelques salariés de la commission scolaire.

Le contrat intervenu entre un tel entrepreneur et la commission scolaire comprenait notamment ces modalités:

- en cas d'ambiguïté, l'interprétation de l'employeur devait lier le cocontractant;

- le travail à exécuter répondait d'un devis «descriptif fort détaillé» (para. 2.18);

- l'exécution était faite sous la surveillance du directeur de l'école et selon l'horaire qu'il fixe;

- ce co-contractant était rémunéré en raison de 26 versements égaux;

- le co-contractant était personnellement lié par ce contrat et ne pouvait le céder, le transférer et ni le résilier: le décès seul pouvait le rompre;

1 La Commission scolaire du Lac Témiscamingue c. le Syndicat canadien de la fonction publique, 1986 TT 106.

Relat. ind., vol. 41, no 4, 1986 (C) PUL ISSN 0034-379 X 
- le donneur d'ouvrage pouvait cependant résilier le contrat à la suite d'un préavis, s'il n'était pas satisfait du travail exécuté;

- le co-contractant était libre d'engager des salariés pour effectuer le travail.

Notons que la commission scolaire exigeait que le co-contractant forme d'abord une corporation bien qu'elle en assumait les frais et demandait que l'actionnaire dispose du contrôle majoritaire du capital-actions et s'engage à ne pas le céder sans autorisasion préalable de la commission

\section{LES MOTIFS DU TRIBUNAL}

Les premières remarques du juge permettaient de croire à une autre orientation que celle qui fut effectivement retenue. En effet, on pouvait lire les observations suivantes:

\section{L'obligation corporative}

«La commission scolaire décida d'exiger l'incorporation de ceux-ci après qu'elle eut reçu en 1983 une requête de l'intimé en vertu de l'article 39 du Code concernant 12 détenteurs de contrat» (page 109).

\section{L'appréciation du commissaire}

On y reproduit de larges extraits de la décision de première instance où le commissaire disait:

«Les entrepreneurs ménagers font exactement le même travail que les concierges syndiqués ou ouvriers spécialisés dans quelques cas. Les premiers remplacent les seconds. La nature de leur travail est donc la même. (...) Les matériaux et l'outillage sont fournis par la commission. (...) La plupart du temps ces entrepreneurs se font aider par des membres de leur famille ce qui lui permet de soumissionner à plus bas prix et assure à la commission une économie de $\$ 100000$ par an. (...) L'analyse du devis descriptif démontre que la commission en plus de cédules de travail de l'entrepreneur lui dit quoi faire en détail chaque jour, chaque semaine, chaque mois». (page 110)

Après avoir rapporté les deux thèses en présence, le Tribunal du travail répond à la question relative au fardeau de la preuve concernant la qualification de salarié. Parce que la commission scolaire n'invoquait pas une exception de la définition de salarié donnée au Code du travail, mais contestait de plein fouet la qualification même de salarié, il revenait, soutient-on, au syndicat de le démontrer:

«Je pense même que si prima facie l'on est en présence d'un entrepreneur, il reviendra à celui qui soutient le contraire d'en faire la démonstration».

Au sujet de la relation du co-contractant ou donneur d'ouvrage, le juge considère que ce dernier n'avait pas pleine discrétion pour résilier le contrat: 
«Il faut que le travail ne soit pas exécuté conformément aux exigences du contrat, et il s'agit là d'une question de faits, non pas d'appréciation subjective, pour qu'il puisse y avoir résiliation en vertu de la clause». (page 111)

Et il ajoute que le directeur de l'école ne donnait pas de directives et que s'il connaissait des difficultés, ce sont les gestionnaires de la commission scolaire qui devaient s'en charger:

«Le directeur d'école leur fait parfois savoir qu'il y a telle ou telle chose à faire mais ni l'un ni l'autre n'a mentionné un seul incident où l'on devrait qualifier d'ordre ce type d'information-demande de la part du directeur d'école». (page 111)

Quant à la question du maintien et du contrôle de la corporation qu'exigeait la commission scolaire, le juge la considère normale, dans les circonstances.

«S'agissant ici d'un milieu scolaire, il n'est guère surprenant non plus que l'on veuille pouvoir refuser un actionnaire principal autre que celui qui était en place lors de l'octroi du contrat; M. Bastien nous a dit que des soumissionnaires avaient déjà été refusés pour des raisons d'ordre moral». (page 111).

Finalement, le tribunal énonce ainsi son principal motif:

«Au niveau du vécu, le preuve fait voir que les détenteurs de contrat possèdent l'autonomie caractéristique de l'entrepreneur. Sous réserve de ne pas nuire aux activités à l'école, ce qui est le bon sens même, ils décident du temps où ils feront les travaux prévus; ils sont maîtres de la façon d'exécuter ces travaux; ils ne sont pas tenus d'exécuter eux-mêmes les travaux et, dans les faits, certains d'entre eux embauchent du personnel (Ewen a quatre employés réguliers et quelques temps partiels; il s'agit toutefois du cas extrême). Ce dernier élément est d'importance primordiale; je fais miens à cet égard les propos suivants de mon collègue Bernard Lesage dans l'affaire Gaston Breton Inc... Je crois que les aspects contractuels des relations entre les parties examinés dans le vécu, doivent primer sur les aspects économiques de celles-ci; le rôle de ces derniers aspects se limite à aider, au besoin, à mieux cerner la réalité contractuelle». (page 112)

\section{COMMENTAIRES}

Au premier abord, cette affaire apparaît être une version travailliste de la célèbre décision en droit des corporations ${ }^{2}$ où on sut bien distinguer la personne de l'actionnaire même solitaire de la corporation dont il était la seule âme dirigeante. Si un tel dédoublement fut vital pour l'essor du droit des corporations, la portée de cette décision pourrait être désastreuse en droit du travail. Cette affaire permet également une autre analogie: celle de la décision J.J. Joubert de 1965 (D.C.D.R.T. no 1638-10) où la C.R.T. reconnut la mutation que l'employeur d'alors imposa à ses vendeurssalariés pour en faire des vendeurs-entrepreneurs. Non seulement les effets recherchés par les initiateurs patronaux sont semblables en ces deux affaires, mais la démarche empruntée, vingt-et-un ans après, est la même: 
-- le test de la fourchette à quatre dents: contrôle, propriété des instruments de travail, possibilité de gains et risque de pertes;

- le dispositif est de même portée soit le non-assujettissement au Code du travail.

Cet emprunt est d'autant plus surprenant que la Cour supérieure avait pourtant mis à découvert en 1972 la vraie nature de cette relation de travail au-delà de son maquillage ${ }^{3}$.

La question la plus intéressante ou peut-être, la plus intrigante consistait à mieux connaître les motifs de l'employeur:

i) pour procéder à l'appel d'offre au lieu d'effectuer de nouveaux engagements dès le départ d'un salarié-concierge:

ii) pour imposer l'incorporation au co-contractant;

iii) pour payer les frais de formation de la corporation;

iv) pour surveiller le maintien du contrôle majoritaire par l'actionnaireconcierge.

On ne trouve guère d'éléments de réponses à cette complexe question et pourtant, elle nous paraît révélatrice du double effect recherché: se défaire du syndicat et réduire les frais d'entretien ménager par l'effet d'une concurrence entre des corporations-concierges. Au sujet de ces profits et pertes, on ignore aussi si l'économie de $\$ 100000$, dont l'employeur pourrait bénéficier, tient compte des frais de formation de ces corporations, des frais annuels encourus au plan comptable et administratif pour la survie de la corporation et des frais de leur liquidation éventuelle?

Si telle est la «réalité contractuelle» des parties au sens de ce jugement et si la commission scolaire a effectivement confié ses travaux ménagers à des entrepreneurs alors, ne leur faudrait-il pas appliquer intégralement le Code du travail? Nous devons alors retenir que ces mêmes activités sont visées par une accréditation et que les salariés-concierges qui y demeurent sont assujettis à une convention collective. Alors, ces corporationsconcierges ne sont-elles pas de nouveaux employeurs au sens et pour les fins de l'art. $45 \mathrm{Ct}$ ? Rien ne s'oppose d'ailleurs à ce que des conciergesactionnaires soient salariés ${ }^{4}$. De plus, si quelques difficultés pratiques surgissaient des effets immédiats de l'art. $45 \mathrm{Ct}$, on sait que le commissaire du travail puis le Tribunal du travail peuvent fournir les indications nécessaires pour les aplanir (art. $46 \mathrm{Ct}$ ).

Par ailleurs, le juge soutient que sa décision suit l'enseignement d'une autre décision du Tribunal dans l'affaire Gaston Breton'. Il nous faut rappeler que dans cette dernière affaire, le livreur-vendeur retenait aussi les services d'aide et que ce fait ne lui fit pas perdre la qualité de salarié.

Au-delà de cette «réalité contractuelle» au sens de ce jugement, nous sommes assez surpris qu'un entrepreneur puisse être aussi peu libre:

3 J.J. Joubert c. Lapierre, 1972, C.S. 476.

4 Fri Information Services Ltd., 1982, C.S. 742.

5 1980, T.T. 471. Voir aussi une autre façon d'effectuer la qualification du statut d'un concierge: Caisse Populaire St-Malo c. Syndicat des travailleuses de la Caisse, 1986, T.T. 113. 
- de sa personne corporative: la cession des actions est soumise au contrôle du donneur d'ouvrage;

- de son contrat d'entreprise: il ne peut ni le céder ni le rompre et il doit directement en assumer l'exécution;

- de son horaire de travail;

- du fait qu'il soit payé en vingt-six versements comme les conciergessalariés.

C'est à douter que ces «corporations-concierges» exerçaient vraiment leurs activités comme s'ils étaient vraiment des «propriétaires d'entreprises» selon l'expression déjà retenue par le tribunal ${ }^{6}$. Cette approche pour le moins formaliste c'est-à-dire qui donne plus d'importance à la fiction juridique qu'à la réalité concrète, constitue un encouragement à utiliser pareil subterfuge. Pourtant, il y a déjà vingt ans, la Cour suprême du Canada avait déjà mis à découvert l'écran juridique pour reconnaître le véritable employeur?

6 Syndicat des employés des Publications Québécor et Publications Québécor Inc., 1977, T.T. 46.

7 Bakery and Confectionery Workers Int. Union and White Lunch, 1966, R.C.S 282, commenté à (1967) 22 R.I. 278.

\section{Travail Labour \\ Canada Canada}

Le Comité syndical-patronal de l'industrie canadienne des textiles: la qualité de la vie au travail, Collection «Elude de cas», par Jean Sexton, Claudine Leclerc et Michel Audet. Nouvelle parution, 1985. L'histoire, le fonctionnement et les travaux d'un comité syndical-patronal. L46-1548/85F ISBN 0-662-93188-2 108 pp.

The Canadian Textile Labour-Management Committee: Quality of Working Life Case Studies Series, by Jean Sexton, Claudine Leclerc, and Michel Audet. The history, structure and achievements of a sector-wide labour-management committer. New. 1985. L46-1548/85E ISBN 0-662-13985-5 94 pp.

$$
\text { Distribution gratuite - Available free of charge }
$$

TRAVAII. CANADA

Centre de distribution des publications

\section{LABOUR CANADA}

Publication Distribution Centre

OTTAWA, ONTARIO

K 1 A 0 J 2 